SHORT REPORT

\title{
Similarity and disparity of obsessive-compulsive disorder and schizophrenia in MR volumetric abnormalities of the hippocampus-amygdala complex
}

\author{
J S Kwon, Y-W Shin, C-W Kim, Y I Kim, T Youn, M H Han, K-H Chang, J-J Kim
}

Objectives: Given that obsessive-compulsive disorder (OCD) and schizophrenia may share clinical symptoms as well as functional brain abnormalities, this study was designed to clarify common and different morphological abnormalities in OCD and schizophrenia.

Methods: Volumes of the hippocampus, the amygdala, and the thalamus were measured in three age and sex matched groups of 22 patients with OCD, 22 patients with schizophrenia, and 22 normal subjects using three dimensional magnetic resonance imaging. Volume tracing was performed manually on serial coronal slices with the references of sagittal or axial planes using internal landmarks. Results: Hippocampal volume was bilaterally reduced in both OCD and schizophrenic patients versus the normal controls. Left amygdala volume was significantly enlarged in patients with OCD but not in patients with schizophrenia versus the normal controls. The thalamus did not show any volumetric group differences.

Conclusions: Non-specific hippocampal reduction in both the OCD and schizophrenic groups is likely to link to a clinical overlap between the two illnesses, whereas the left amygdala enlargement observed only in the OCD patients seems to be suggestive of a unique role for the amygdala in the pathophysiology of OCD.

A lthough OCD and schizophrenia are separate psychiatric disorders, patients having comorbidities of the two illnesses are frequently found in clinical practice. Based on functional and structural neuroimaging findings, the possible overlap has been also identified in the pathophysiology of the two illnesses, in particular abnormalities in the frontostriatal circuits. ${ }^{1}$ Other candidate structures for such overlapping pathophysiology may include the thalamus and/or the hippocampus-amygdala complex. It is noteworthy that although similar regions have been identified to be functionally or morphologically abnormal in both illnesses, they tend to occur in opposite directions: for example, hyperfunctional fronto-striatal systems in $\mathrm{OCD},{ }^{2}$ but hypofunctional in schizophrenia ${ }^{3}$; and thalamic enlargement in $\mathrm{OCD}^{4}{ }^{4}$ but thalamic reduction in schizophrenia. ${ }^{5}$

Based on such significant but different findings of similar regions in OCD and schizophrenia, we hypothesised that the two illnesses would also show quite different abnormal patterns in the hippocampus-amygdala complex. Moreover, it was expected that such morphological changes, if identified, would correspond to hyperfunctional circuitry in OCD, but hypofunctional circuitry in schizophrenia. To test this hypothesis, volumes of the hippocampus, the amygdala, and the thalamus were compared in patients with OCD, in patients with schizophrenia, and in normal subjects.

\begin{abstract}
METHODS
Subjects

There were three age and sex matched groups: OCD, schizophrenia, and normal subjects. Each group consisted of 22 subjects ( 15 men and 7 women), who were all right handed, and had a mean age of 26.7 (SD 7.2), 26.6 (SD 6.5), and 26.2 (SD 6.1) years, respectively. Patients were recruited from Seoul National University Hospital, and none had a dual diagnosis of OCD and schizophrenia. Normal volunteers were recruited from the community through newspaper advertisements. Mean period of education and IQ were statistically different among the groups, but mean parental socioeconomic status was not significantly different. This study was carried out under guidelines for the use of human subjects established by the institutional review board. Written informed consent was obtained from all subjects.
\end{abstract}

\section{Magnetic resonance image acquisition and processing} Three dimensional Tl weighted spoiled gradient echo MR images were acquired on a 1.5 tesla GE SIGNA scanner, using imaging parameters of $1.5 \mathrm{~mm}$ sagittal slices, $\mathrm{TE}=5.5 \mathrm{~ms}$, TR $=14.4 \mathrm{~ms}, \mathrm{NEX}=1$, rotation angle $=20^{\circ}, \mathrm{FOV}=21 \times 21 \mathrm{~cm}$, and a matrix of $256 \times 256$. MR images were processed using an image processing software package, ANALYZE (version 3.0, Mayo Foundation, USA). Images were resampled, reoriented, spatially realigned to the conventional position, and filtered using the anisotropic diffusion method with five iterations to improve the signal to noise ratio. Intracranial volume (ICV) was calculated after the brain extraction and segmentation.

\section{ROI tracing}

The neuroanatomical regions of interest (ROIs) were traced manually on all coronal slices with the references of sagittal or axial planes using the ANALYZE ROI module. Boundaries of the hippocampus were defined using a minor modification of the method previously reported by Pantel and colleagues. ${ }^{6}$ Tracing was performed initially on the sagittal slices for a reliable separation, and proceeded to the coronal plane applying different boundaries according to the positions of the head, body, or tail of the hippocampus. Boundaries of the amygdala were preliminarily drawn on the axial plane, using a modification of the delineating methods of Honeycutt and colleagues $^{7}$ and Convit and colleagues ${ }^{8}$; the main tracing was performed on the coronal view. Boundaries of the thalamus were based on the detailed guidelines of Portas and colleagues. ${ }^{9}$ The medial and lateral geniculate bodies were included as a part of the thalamus.

In order to assess inter-rater reliability, a tracing was performed independently on a set of 10 MR scans by two

Abbreviations: ICV, intracranial volume; $M R$, magnetic resonance; $\mathrm{OCD}$, obsessive-compulsive disorder; ROI, region of interest 
Table 1 Absolute volumes $(\mathrm{ml})$ of the three regions of interest ${ }^{*}$

\begin{tabular}{llllll}
\hline Region & $\begin{array}{l}\text { Normal } \\
(n=22)\end{array}$ & $\begin{array}{l}\text { OCD } \\
(n=22)\end{array}$ & $\begin{array}{l}\text { Schizophrenia } \\
(n=22)\end{array}$ & $F$ & $p$ \\
\hline $\begin{array}{l}\text { Hippocampus } \\
\text { Left }\end{array}$ & $3.21(0.33)$ & $2.98(0.38) \dagger$ & $2.87(0.42) \dagger$ & 6.92 & $<0.01$ \\
$\quad \begin{array}{l}\text { Right } \\
\text { Amygdala }\end{array}$ & $3.10(0.31)$ & $2.90(0.30) \dagger$ & $2.85(0.40) \dagger$ & 6.29 & $<0.01$ \\
$\quad$ Left & & & & & \\
$\quad$ Right & $0.58(0.14)$ & $0.71(0.15) \ddagger$ & $0.62(0.11)$ & 4.17 & 0.02 \\
$\quad \begin{array}{l}\text { Thalamus } \\
\text { Left }\end{array}$ & $0.67(0.16)$ & $0.79(0.18)$ & $0.69(0.10$ & 2.33 & 0.11 \\
$\quad$ Right & $5.65(0.56)$ & $5.92(0.69)$ & $5.80(0.70)$ & 0.11 & 0.89 \\
Intracranial volume & $5.66(0.64)$ & $5.90(0.72)$ & $5.69(0.53)$ & 0.25 & 0.77 \\
\hline & $1354(98)$ & $1420(134)$ & $1386(114)$ & 1.77 & 0.18 \\
\hline
\end{tabular}

*Data are given as mean (SD). Statistical significance levels are based on one way ANCOVA.

†Post hoc tests indicated smaller left and right hippocampal volumes in both the schizophrenia and OCD groups than in the normal comparison group.

$\ddagger$ Post hoc tests indicated greater left amygdala volume in the OCD group than in the schizophrenia and comparison groups.

raters (YIK and CWK) and volumes of the traced regions were measured automatically. The intra-class correlation coefficients (ICC) were calculated and found to be high: 0.89/0.90, $0.87 / 0.91$, and $0.95 / 0.97$ for the left/right side of the hippocampus, amygdala, and thalamus, respectively. These high reliability scores corresponded to relatively small percentage volume differences - that is, the smallest $1.8 \%$ (SD 1.1) for the right thalamus and the largest 6.7\% (SD 5.3) for the left amygdala. In the case of the between group measures, one rater (YIK) traced the hippocampus and the amygdala, and the other rater (CWK) traced the thalamus.

\section{Statistical analysis}

Primary analysis was performed using a mixed model ANCOVA with one between subjects factor and one within subjects factor for each region. This analysis was followed by one way ANCOVA with Bonferroni post hoc tests for the left and right of each individual. One way ANOVA was used to test for ICV group differences. The ICV was used as covariates for all ANCOVAs. All statistical analyses were two tailed, and $\mathrm{p}<0.05$ was used as a level of significance.

\section{RESULTS}

As presented in table 1, mean ICV was not statistically different among the three groups. A significant main group effect was found for hippocampal volumes $\left(\mathrm{F}_{2,63}=7.30, \mathrm{p}<0.01\right)$, and significant group differences were revealed in both sides of the hippocampus (left: $\mathrm{F}_{2,63}=6.92, \mathrm{p}<0.01$; right: $\mathrm{F}_{2,63}=$ $6.29, \mathrm{p}<0.01)$; both OCD and schizophrenia groups had bilaterally smaller hippocampal volumes than the normal comparison subjects. No significant main effect of side, and interaction effect of side-by-group were found.

A significant main group effect was also found for amygdala volumes $\left(\mathrm{F}_{2,63}=3.50, \mathrm{p}<0.05\right)$. A significant group difference was revealed in the left amygdala only $\left(\mathrm{F}_{2,63}=4.17, \mathrm{p}<0.05\right)$; the OCD group had a greater left amygdala volume than both the schizophrenia and the normal groups. No significant main effect of side, and interaction effect of side-by-group were found. In case of thalamic volume, any significance was not found in a main group effect, group differences for each side, and a main effect of side and interaction effect of side-bygroup.

\section{DISCUSSION}

Our findings of a bilaterally smaller hippocampus in both OCD and schizophrenia than in normal subjects show that hippocampal volume reduction is not specific to each illness. Although hippocampal volume reduction has been one of the most consistent abnormal morphological findings in schizophrenia, a comparison of schizophrenia with affective psychosis also fails to show its specificity. ${ }^{10}$ Therefore, these non-specific findings are likely to link to clinical overlap among the psychiatric illnesses, although its mechanism is unclear.

Contrary to the hippocampus, findings from the amygdala support our hypothesis that OCD and schizophrenia reveal different abnormal patterns. Enlargement of the left amygdala in OCD may relate to amygdala hyperfunction, and supports the amygdalocentric model regarding the pathophysiology of OCD. ${ }^{11}$ The amygdala may be a key neuroanatomic substrate of the anxiety that perpetuates compulsions in OCD. Given that the anxiety mediated by amygdala is inhibited by feedback from the orbitofrontal cortex, ${ }^{12}$ the normal cortical-toamygdala inhibition that prevents the amygdala from being overactive is likely to malfunction in OCD.

Although the thalamic volume did not show any group differences, it may be too large as an ROI to detect subtle changes confined to a specific thalamic nucleus. Significant thalamic abnormalities in schizophrenia have only emerged when single specific thalamic nuclei have been considered. ${ }^{5}$ Subregional thalamic abnormality in OCD was found in our previous study ${ }^{4}$ using a voxel based morphometric approach, and was too small to detect using the present ROI method. Therefore, a subregional approach is needed to identify a possible thalamic abnormality in OCD, as attempted in schizophrenia.

To our knowledge, this is the first report that compares volume measures of the hippocampus-amygdala complex in OCD and schizophrenia. Further research to determine the nature of the different involvements of OCD and schizophrenia with the abnormal fronto-temporal network may shed further light on the pathophysiology of these illnesses.

\section{ACKNOWLEDGEMENT}

This work was supported by Korea Research Foundation Grant (KRF2001-044-F00182).

\section{Authors' affiliations}

J S Kwon, Y-W Shin, C-W Kim, Y I Kim, T Youn, Department of Psychiatry, Seoul National University College of Medicine, Seoul, Korea J-J Kim, BK2 1 Life Science, Seoul National University College of Medicine

M H Han, K-H Chang, Department of Radiology, Seoul National University College of Medicine

Conflict of interest: None declared

Correspondence to: Dr J-J Kim, BK21 Life Sciences, Room 206, Seoul National Univeristy College of Medicine, 28 Yeongon-dong, Chongno-gu, Seoul, Korea, 11 10-744; ijkim@neuroimage.snu.ac.kr

Received 16 April 2002

In revised form 18 September 2002

Accepted 13 December 2002 


\section{REFERENCES}

1 Cummings JL. Frontal-subcortical circuits and human behavior. Arch Neurol 1993:50:873--80.

2 Baxter LR, Schwartz JM, Mazziotta JC, et al. Cerebral glucose metabolic rates in nondepressed patients with obsessive-compulsive disorder. Am J Psychiatry 1988;145:1560-3.

$3 \mathrm{Kim}$ JJ, Mohamed S, Andreasen NC, et al. Regional neural dysfunctions in chronic schizophrenia studied with positron emission tomography. Am J Psychiatry 2000;157:542-8.

$4 \mathrm{Kim}$ JJ, Lee MC, Kim JS, et al. Grey matter abnormalities in obsessive-compulsive disorder: statistical parametric mapping of segmented magnetic resonance images. Br J Psychiatry 2001;179:330-4.

5 Andreasen NC, Arndt S, Swayze V 2nd, et al. Thalamic abnormalities in schizophrenia visualized through magnetic resonance image averaging. Science 1994;266:294-8.

6 Pantel J, O'Leary DS, Cretsinger K, et al. A new method for the in vivo volumetric measurement of the human hippocampus with high neuroanatomical accuracy. Hippocampus 2000;10:752-8.
7 Honeycutt NA, Smith PD, Aylward E, et al. Mesial temporal lobe measurements on magnetic resonance imaging scans. Psychiatry Res 1998;83:85-94.

8 Convit A, McHugh P, Wolf OT, et al. MRI volume of the amygdala: a reliable method allowing separation from the hippocampal formation. Psychiatry Res 1999:90:113-23.

9 Portas CM, Goldstein JM, Shenton ME, et al. Volumetric evaluation of the thalamus in schizophrenic male patients using magnetic resonance imaging. Biol Psychiatry 1998;43:649-59.

10 Hirayasu Y, Shenton ME, Salisbury DF, et al. Lower left temporal lobe MRI volumes in patients with first-episode schizophrenia compared with psychotic patients with first-episode affective disorder and normal subjects. Am J Psychiatry 1998:155:1384-91.

11 Rauch SL, Whalen PJ, Dougherty D, et al. Neurobiologic models of obsessive-compulsive disorder. In: Jenike MA, Baer L, Minichiello WE, eds. Obsessive-compulsive disorders: practical management, 3rd edn. St Louis: Mosby, 1998:222-53.

12 Morgan MA, LeDoux JE. Differential contribution of dorsal and ventral medial prefrontal cortex to the acquisition and extinction of conditioned fear in rats. Behav Neurosci 1995;109:681-8.

\section{$\mathrm{ECHO}$}

\section{Population study extends profile of Rett syndrome}

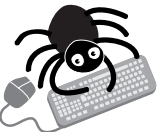

Please visit the Journal of Neurology, Neurosurgery and Psychiatry website [www.jnnp.com] for link to this full article. $\square$ he first genetically described population based study in Rett syndrome-a childhood developmental disorder-has provided a more complete clinical picture to enable correct diagnosis and prognostication. It provides invaluable information for future studies on genotype-phenotype interactions too.

The study used phenotypic data from the Australian Rett Syndrome Database population registry from 1993 onwards, supplemented with information from a follow up questionnaire to each family in the first half of 2000. Blood samples were taken from the children to test for MECP2 gene mutation. The phenotypic data were used to describe variation in the syndrome according to different severity scales for Rett syndrome-Kerr, Percy, and Pineda scales-and to calculate WeeFIM (Functional Independence Measure for Children) score.

In all, 161 verified cases were eligible for the study. The families of 152 affected children responded to the questionnaire. The researchers judged $55 \%$ to be classic cases, $26 \%$ early onset atypical cases, and 19\% mild atypical cases. The proportion of classic cases in the oldest group was almost twice that of the youngest ( $73 \% v 42 \%)$. Results of genetic screening were available for 134 (88\%) of the sample and showed MECP2 mutation in $72 \%$ : 73\% in classic, $62 \%$ early onset atypical, and $80 \%$ mild atypical cases. The four scales provided detailed information on severity and how age changed some criteria.

Rett syndrome results in impaired development usually starting about age 6 months. Population based research, say the researchers, "is still, after nearly 20 years, not yet informing treatment or intervention for Rett patients."

\ Archives of Disease in Childhood 2003;88:38-43. 